\title{
Genetic screening in sudden cardiac death in the young can save future lives
}

\author{
Eva-Lena Stattin $^{1}$ (D) - Ida Maria Westin ${ }^{1} \cdot$ Kristina Cederquist $^{1} \cdot$ Jenni Jonasson $^{1}$ • \\ Björn-Anders Jonsson ${ }^{1}$ • Stellan Mörner ${ }^{2}$ - Anna Norberg ${ }^{1}$ • Peter Krantz ${ }^{3}$. \\ Aase Wisten ${ }^{4}$
}

Received: 16 October 2014 / Accepted: 16 July 2015 / Published online: 31 July 2015

(C) The Author(s) 2015. This article is published with open access at Springerlink.com

\begin{abstract}
Background Autopsy of sudden cardiac death (SCD) in the young shows a structurally and histologically normal heart in about one third of cases. Sudden death in these cases is believed to be attributed in a high percentage to inherited arrhythmogenic diseases. The purpose of this study was to investigate the value of performing post-mortem genetic analysis for autopsy-negative sudden unexplained death (SUD) in 1 to 35 year olds.

Methods and results From January 2009 to December 2011, samples from 15 cases suffering SUD were referred to the Department of Clinical Genetics, Umeå University Hospital, Sweden, for molecular genetic evaluation. PCR and bidirectional Sanger sequencing of genes important for long QT syndrome (LQTS), short QT syndrome (SQTS), Brugada syndrome type $1(\mathrm{BrS1})$, and catecholaminergic polymorphic ventricular tachycardia (CPVT) (KCNQ1, KCNH2, SCN5A, $K C N E 1, K C N E 2$, and $R Y R 2$ ) was performed. Multiplex ligation-dependent probe amplification (MLPA) was used to detect large deletions or duplications in the LQTS genes. Six pathogenic sequence variants (four LQTS and two CPVT) were discovered in 15 SUD cases $(40 \%)$. Ten first-degree family members were found to be mutation carriers (seven LQTS and three CPVT).
\end{abstract}

Eva-Lena Stattin

eva-lena.stattin@umu.se

1 Department of Medical Biosciences, Medical and Clinical Genetics, Umeå University, Umeå, Sweden

2 Heart Centre and Department of Public Health and Clinical Medicine, Umeå University, Umeå, Sweden

3 Department of Forensic Medicine, Lund University, Lund, Sweden

4 Department of Internal Medicine, Sunderby Hospital, Luleå, Sweden
Conclusion Cardiac ion channel genetic testing in autopsynegative sudden death victims has a high diagnostic yield, with identification of the disease in $40 \%$ of families. Firstdegree family members should be offered predictive testing, clinical evaluation, and treatment with the ultimate goal to prevent sudden death.

Keywords Sudden unexplained death $\cdot$ Sudden cardiac death · Molecular autopsy $\cdot$ Long QT syndrome .

Catecholaminergic polymorphic ventricular tachycardia
Abbreviations
BrS Brugada syndrome
CPVT Catecholaminergic polymorphic ventricular tachycardia
LQTS Long QT syndrome
MLPA Multiplex ligation-dependent probe amplification
SCD Sudden cardiac death
SUD Sudden unexplained death
SQTS Short QT syndrome

\section{Introduction}

Sudden cardiac death (SCD) in the young is a tragic event, with an incidence of 1-2 per 100,000 per year, in the age group 15 to 35 years old [1-4]. SCD is defined as a witnessed, natural unexpected death from cardiac causes occurring within $1 \mathrm{~h}$ after onset of symptoms in a previously healthy person or an unwitnessed natural unexpected death of a person observed to be well within $24 \mathrm{~h}$ of being found dead [5]. Standard forensic autopsy is often unsuccessful in determining the causes of sudden death $[1,6-8]$. Negative toxicology and a structurally and histologically normal heart is found in up to 
$53 \%$ of these cases, commonly referred to as autopsy-negative, sudden unexplained death (SUD) $[1,3,9]$. There are several conditions difficult to discover at autopsy that may cause SUD. Inherited cardiac diseases such as channelopathies and cardiomyopathies are a plausible cause of such autopsy-negative SCD cases and may explain a significant number of these events [10-13]. Studies have shown that genetic testing may identify a cardiovascular cause of death in up to $30 \%$ of these cases [11, 14-19]. Long QT syndrome (LQTS), short QT syndrome (SQTS), Brugada syndrome $(\mathrm{BrS})$, and catecholaminergic polymorphic ventricular tachycardia (CPVT) causing isolated electric dysfunction are due to defective genes encoding subunits of cardiac ion channels $\left(\mathrm{K}^{+}, \mathrm{Na}^{+}\right.$, or $\left.\mathrm{Ca}^{2+}\right)$ or ion channel regulatory proteins $[20$, 21]. Disturbed ion flow is the substrate to ventricular arrhythmias causing syncope and increased risk of SCD, which characterize these disorders. Syncope is the most common symptom in ion channel disorders, although cardiac arrest may be the first clinical manifestation in apparently healthy subjects. In LQTS, death is the first symptom of the disease in 10-15\% of cases, [21] whereas in CPVT, death can be the first clinical presentation in up to $30 \%$ of cases [22]. The mean penetrance of pathogenic RYR 2 variants causing CPVT is $83 \%$, with mortality rates of $30-50 \%$ by age 35 years, and consequently, asymptomatic carriers are rare [20,22]. It is also useful to test for cardiomyopathies such as hypertrophic cardiomyopathy (HCM) and arrhythmogenic right ventricular cardiomyopathy (ARVC) as structural changes in the cardiac tissue connected with these diseases can be difficult to discover at autopsy [17]. In this nationwide prospective study, our first aim was to determine the value of molecular genetic autopsy in young SUD victims by genetic screening of genes important for LQTS, SQTS, BrS1, and CPVT. Mutation carriers are often unaware of their disease, and unfortunately, sudden death in many cases is the first chance to reveal the disorder. Molecular autopsy can give an explanation to the sudden death, which is important for the mourning process of the family [23]. The findings of an inherited genetic disorder also provide an opportunity to offer first-degree family members cascade screening; identification of mutation carriers can save future lives $[16,24,25]$. Our second aim was to offer first-degree relatives genetic testing and medical surveillance to mutation carriers.

\section{Methods}

\section{Inclusion criteria}

Between January 2009 and December 2011, the Swedish departments of forensic medicine were invited to send whole blood in EDTA tubes and tissue samples from cases of SUD. Toxicological analysis including illicit drugs and narcotics was performed in all cases. Histological examination of the heart were performed, comprising both the left and right ventricle, and in some cases also the conduction system, the average number of samples taken from the heart was 11 with a range from 3 (one case) to 26. The specimens were examined by trained investigators in haematoxilin-eosin staining as well as PTAH staining. To be included in the study, the deceased had to be $1-35$ years old and the death had to be sudden, unexpected, and unexplained after comprehensive medicolegal autopsy. Unnatural causes of death such as trauma, murder, suicide, or intoxication were excluded. In total, 15 SUD victims ( 10 male and 5 female, medium age at death 15 years) fulfilled these criteria and were included in the study.

\section{Clinical data}

Data on included SUD victims were collected from forensic autopsy reports and police reports. Information on preceding symptoms and medical history of the deceased was assembled from these sources supplemented with interviews with firstdegree relatives and medical records when available.

\section{Genetic analyses}

The genetic analyses and interpretation of pathogenicity of sequence variants have been performed as part of ordinary health care diagnostics at a clinical genetics laboratory experienced with sequence analysis of ion channel disorders. DNA was extracted from blood or tissue samples using standard salting-out methods. DNA were analyzed for sequence variants in all protein-coding exons and their flanking splice site regions of the genes KCNQ1 (NM_000218.2 and NM_181798.1), KCNH2 (NM_000238.2 and NM_172057.1), SCN5A (NM_198056.1), KCNE1 (NM_000219.2), and KCNE2 (NM_172201.1) using PCR and bidirectional sequencing on an ABI3500 Genetic Analyzer (Applied Biosystems, Foster City, CA, USA). DNA were also analyzed for sequence variants in the functionally most important exons (8-15, 44-50, 83, 88-105) of the gene RYR2 (NM_001035.2). Two cases with pathogenic sequence variants in the $K C N Q 1$ gene were only screened for sequence variants in the KCNQ1 gene. Primers were M13-tagged and modified from Jongbloed et al. or designed with Primer3 software (primer sequences are available upon request). To detect deletions or duplications, five samples were analyzed using multiplex ligation-dependent probe amplification (MLPA) and the SALSA P114-A2 kit (MRC-Holland). This kit covers exons $1 \mathrm{~B}, 1-4,6-13$, and $15-16$ of $K C N Q 1$, exons $1 \mathrm{~B}, 1-4,6$, 9-10, and 14 of $K C N H 2$, exons 1-2 of $K C N E 2$, exons 2-4 of $K C N E 1$, and exons 2, 4, 25, and 27 of SCN5A. Direct DNA sequencing of the protein coding exon was used for cascade screening of family members. 


\section{Interpretation of identified sequence variants}

Sequences were evaluated using the Sequencer software version 4.9 (Gene Codes Corporation, MI, USA) and compared with a reference genomic sequence. All identified variants were denoted using accepted nomenclature recommended by the Human Genome Variation Society. In silico predictions were made for all identified sequence variants using the Alamut software version 2.2 (Interactive Biosoftware, Rouen, France). The Alamut software assists in evaluation of missense variants by compiling output from a number of bioinformatic prediction tools, including PolyPhen, SIFT, AlignGVGD, and MutationTaster [26-28]. Novel missense variants were tested for presence in 200 control chromosomes of the same population and checked for presence in the 6500 control exomes of the NHLBI Exome sequencing project.

\section{Ethics approval}

The Regional Ethical Review Board in Umeå, Sweden, approved this study. All first-degree relatives have given their written informed consent.

\section{Results}

Fifteen SUD victims aged 1-35 years with a structurally normal heart at autopsy were referred for post-mortem ion channel genetic testing, during the period 2009-2011. We estimate that this represent approximately $50 \%$ of SUD cases in this period, according to ongoing investigation of SCD among the young in Sweden 2000-2010 (unpublished data). Toxicological analysis including illicit drugs and narcotics were negative with exception for two cases with therapeutic concentrations of relatively harmless drugs. All cases had normal macroscopic hearts, with the exception of two obese individuals (BMI 33 and 35) who had mild enlargement of their hearts. Histological examination of the left and right ventricle of the heart and in some cases also the conduction system showed no pathological changes that could explain the deaths. Clinical characteristics of the SUD cases are summarized in Table 1. The mean age of death of the SUD victims was $15.5 \pm$ 13 years (mean $\pm \mathrm{SD}$ ), range $2-28$ years. Two of the SUD victims had a family history of sudden death at the time of death. The majority of the subjects, 9/15 (60\%) died during rest or sleep. Two subjects $(13 \%)$ died during physical activity: one after hurrying to the bus stop and one drowned. Two subjects died during daily activity: one sitting in his classroom at school and one died in a car accident. Two subjects died at home without anyone knowing details of the event (Table 1).

\section{Genetic testing of ion channel genes and clinical description of cases}

Genetic screening of the KCNQ1, KCNH2, SCN5A, KCNE1, $K C N E 2$, and RYR2 genes revealed four LQTS and two CPVT mutations $(40 \%)$. There were no deletions or duplications detected with MLPA. None of the pathogenic sequence variants had previously been reported in the literature, dbSNP, or 1000 Genomes, and none were identified in the NHLBI Exome Sequencing Project database (ESP), where $>6000$ European-American and African-American individuals were available. All of the novel variants were predicted to be pathogenic according to at least two bioinformatic tools [26-28]. Characteristics of the identified pathogenic sequence variants are given in Table 2.

\section{Identified LQTS mutations}

Individual $1 \mathrm{~A}$ pathogenic sequence variant in the $K C N Q 1$ gene (Gly220Lys) was identified in a 28-year-old adopted female who was found dead at home during daytime. The woman had given birth to a child 9 months prior to her death. She was depressed, had disturbed sleeping pattern, and was treated with benzodiazepines. She had experienced syncope 2 days prior to the death. The physician at the hospital judged the episode as a vasovagal syncope, and no ECG was taken. The child did not carry the pathogenic sequence variant, and no other family members were available for genetic testing.

Individual 2 A pathogenic sequence variant in the $K C N Q 1$ gene (His240Arg) was identified in an 8-year-old girl who had a cold and died during sleep. Predictive testing of family members revealed three carriers of the pathogenic sequence variant. The mother of the deceased child was found to be a carrier; she was adopted, and nothing is known about her biological parents/siblings. Two siblings were also carriers of the pathogenic sequence variant; one of them has a prolonged QT duration; but none of them have experienced syncope.

Individual $3 \mathrm{~A}$ pathogenic sequence variant in the KCNQ1 gene (Ala370Val) was identified in an 18year-old man who was found dead on the floor at home. Toxicology examinations showed alimemazine and alprazolam in normal therapeutic concentrations in his blood and $1.06 \%$ ethanol in femoral blood. Cascade screening of family members identified four mutation carriers and three none carriers. Two of the carriers have experienced symptoms possible due to LQTS: his grandmother has felt dizziness while swimming a couple of times, and his uncle has experienced dizziness and pre-syncope. 


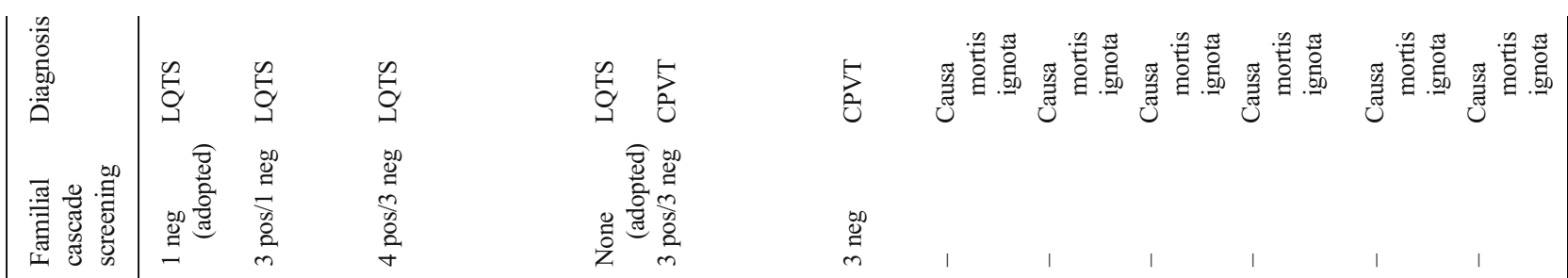

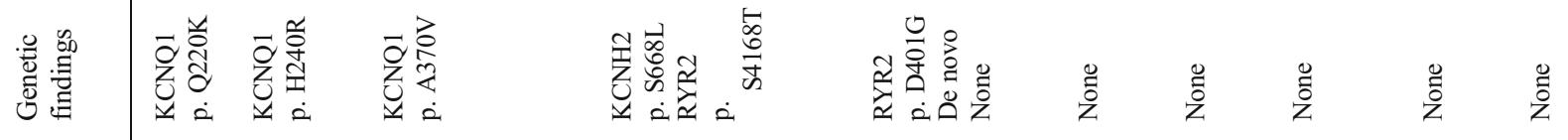


Individual $4 \mathrm{~A}$ pathogenic sequence variant in the $\mathrm{KCNH} 2$ gene (Ser668Leu) was identified in a 14-year-old boy who died sitting at his desk in school. Cardiopulmonary resuscitation was started within minutes but was unsuccessful. The boy was treated with tomoxetine due to attention deficit hyperactivity disorder (ADHD). The boy was adopted; therefore, no genetic investigation of parents or siblings was possible.

\section{Identified CPVT mutations}

Individual $5 \mathrm{~A}$ pathogenic sequence variant in the $R Y R 2$ gene (Ser4168Thr) was identified in an 18-year-old man, who collapsed having hurried to the bus stop. Resuscitation was unsuccessful. The man had been resuscitated 3 years earlier after a similar event and thoroughly investigated without any pathological cardiac findings. He had no current symptoms proceeding death and no treatment. Four weeks after his death, prior to the molecular genetic diagnosis, his younger sister died suddenly when walking back to school after a swimming lesson. She was later found to be a mutation carrier. The sister was born with a tetralogy of Fallot; she had recurrent symptoms of palpitations and syncope. Genetic testing showed that their mother and maternal grandfather also carried the mutation, although neither of them had experienced any symptoms. The maternal great grandfather had experienced syncope and died suddenly unexpected at the age of 46 years, and a maternal grand uncle died suddenly at the age of 64 years; however, we do not know their carrier status. Three family members tested negative for the familial mutation.

Individual $6 \mathrm{~A}$ pathogenic sequence variant in the $R Y R 2$ gene (Asp401Gly) was identified in a 14-year-old boy who drowned, despite that he was a good swimmer. The pathogenic sequence variant was found to be de novo since none of the family members carried the mutation.

In nine of the 15 evaluated cases, we did not identify a disease causing sequence variant; five of them had experienced pre-mortal symptoms, and one had a family history of SUD (Table 1).

\section{Discussion}

This is to our knowledge the first prospective nationwide molecular genetic screening study of the LQTS, SQTS, BrS1, and CVPT genes in SUD victims. Genetic screening of cardiacchannel genes revealed six pathogenic sequence variants (40 \%) among 15 afflicted SUD victims, four LQTS mutations, and two CPVT mutations; ten first-degree family members were found to be carriers and 11 noncarriers. None of the identified mutations had previously been described; thus, all of them were unique to this Swedish cohort. In published LQTS population surveys, most of the mutations are novel 
Table 2 Characteristics of pathogenic sequence variants found in 15 victims of sudden unexplained death aged 1-35 years in Sweden 2009-2011

\begin{tabular}{|c|c|c|c|c|c|c|c|c|c|}
\hline Gene & Exon & Nucleotide change & Amino acid change & Region & Domain & Polyphen- $2^{\mathrm{a}}$ & SIFT & MutationTaster $^{\mathrm{b}}$ & $\begin{array}{l}\text { Align- } \\
\text { GVGD }\end{array}$ \\
\hline \multirow[t]{3}{*}{$K C N Q 1$} & 4 & c. $658 \mathrm{C}>\mathrm{A}$ & p. Gly220Lys & $\mathrm{S} 3-\mathrm{S} 4$ & Ion transport & Possibly damaging & Tolerated & Disease causing & $\mathrm{C} 0$ \\
\hline & 5 & c. $719 \mathrm{~A}>\mathrm{G}$ & p. His240Arg & S4 & Ion transport & Benign & Deleterious & Disease causing & $\mathrm{C} 25$ \\
\hline & 8 & c. $1109 \mathrm{C}>\mathrm{T}$ & p. Ala370Val & C-term & - & Probably damaging & Deleterious & Disease causing & C65 \\
\hline $\mathrm{KCNH} 2$ & 8 & c. $2003 \mathrm{C}>\mathrm{T}$ & p. Ser668Leu & S6 & & Probably damaging & Deleterious & Disease causing & C65 \\
\hline \multirow[t]{2}{*}{$R Y R 2$} & 14 & c. $1202 \mathrm{~A}>\mathrm{G}$ & p. Asp401Gly & N-term & MIR motif & Possibly damaging & Deleterious & Disease causing & C65 \\
\hline & 90 & c. $12502 \mathrm{~T}>\mathrm{A}$ & p. Ser4168Thr & Cytosol & - & Possibly damaging & Deleterious & Disease causing & $\mathrm{C} 55$ \\
\hline
\end{tabular}

SIFT sorting intolerant from tolerant

${ }^{a}$ Polyphen-2, polymorphism phenotyping v2, predicts possible impact of an amino acid substitution on the structure and function of the protein

${ }^{\mathrm{b}}$ MutationTaster calculates probability for the alteration to be either disease causing or a harmless polymorphism

${ }^{\mathrm{c}}$ Align-GVGD Align Grantham variation and Grantham distance, ordered from most likely (C65) to interfere with function to least likely (C0)

( $\approx 60 \%$ ) [29-31]. We have recently described the LQTS mutation panorama in Sweden, and almost one third (28\%) of the identified mutations were novel [32].

\section{Comparison to other studies}

Skinner et al. evaluated the LQT genes in the first prospective population-based series of SUD victims and identified five pathogenic sequence variants in 33 individuals (15\%) [14]. The diagnostic yield concurs with the result in a Danish study but is lower than in our present study, and in the retrospective autopsy studies from the Mayo clinic and from New Zealand $[11,15,33]$. In a Danish cohort of SUD cases, pathogenic sequence variants was identified in five of $44(11 \%)$; cases were tested for sequence variants in the LQT1-3 genes [15]. Tester et al. showed in a retrospective autopsy study that one third (17/49) of young SUD cases had verified genetic causes: LQTS (20\%) or CPVT (15\%) [11]. Gladding et al. used DNA from Guthrie cards collected for newborn metabolic screening and identified disease-causing sequence variants in four of 19 SUD cases (21\%) [33]. The high yield in our present study may be due to sequencing also of the $R Y R 2$ gene, ascertainment bias, or a low number of cases. Recently, Bagnall et al. used exome sequencing of 27 genes and identified nine rare variants in a subset of 28 SUD cases (32\%) [34]. Next generation sequencing and whole exome sequencing will likely be a more effective strategy to uncover the etiology of SUD, although there are limitations and disadvantages of such approaches, including the identification of variants of unknown significance and "co-incidental" genetic pathogenic sequence variants that predispose to adult-onset potentially incurable conditions.

\section{Pre-mortal symptoms are common}

In this study, a man with CPVT had been resuscitated after cardiac arrest 3 years before death, and a woman with LQTS experienced syncope 2 days before she died. The other genotype-positive SUD cases had no reported cardiac-related pre-mortal symptoms. In nine individuals, we did not identify pathogenic sequence variants coding for ion channel disorders; five of these had experienced pre-mortal symptoms such as palpitations or seizures. Medical care was seeked by three of them, but no ECGs were taken. In a Swedish SCD cohort of 15-35 year olds between 1992 and 1999, as many as $46 \%$ had requested medical advice because of symptoms before suffering from SCD [35]. It is possible to identify patients with syncope requiring further evaluation for cardiac etiology [36]. Tretter et al. evaluated syncope in a pediatric setting: using the characteristics in the medical history, physical examination, and ECG, to identify all patients with cardiac etiology [36]. There could be cardiac explanations also to the SUD cases in our study, such as cardiomyopathies with minor visible changes, inflammatory injury of the electrical system in the heart, or pathogenic sequence variants that we were not able to identify. The SUD cases could also have undetected noncardiac background such as epilepsy or drug-related causes.

\section{Activity at time of death}

Syncope usually occurs during exercise or acute emotions, due to torsade de pointes (TdP) in LQTS and due to fast ventricular tachycardia in CPVT [21, 22]. Most often, there is a spontaneous recovery of bidirectional or polymorphic ventricular tachycardia and TdP. However, the arrhythmia may degenerate into ventricular fibrillation, subsequently leading to sudden death if no resuscitation is available [21, 22]. In this study, two out of six carriers of a pathogenic sequence variant died during exercise: one while running (LQTS) and one while swimming (CPVT). Increased risk for death while swimming is recognized both in CPVT and LQTS (especially the LQT1 subtype) [21, 37]. In a postmortem series of drowning victims, pathogenic sequence 
variants in the $R Y R 2$ gene were identified in $20 \%$ [37, 38]. The majority in our study died during normal daily activity or sleep, which is in accordance with other studies [25].

\section{Certain drugs prolong the QT duration}

It is well known that certain drugs prolong the QT duration and might induce TdP in individuals with LQTS [39]. In this study, three of four SUD cases with an identified pathogenic LQT variant were on medication, thus revealing therapeutic levels of medicine in the blood toxicology screening. Both the drugs alimemazine and tomoxetine are known to prolong the QT duration, although the risk for serious cardiovascular adverse events, including significant increases in QTc and sudden cardiac death, is judged to be low. However, great caution is advised when considering stimulant and nonstimulant medications for patients of any age with a diagnosis of ADHD and a personal or family history or other known risk factors for cardiovascular disease [40]. We do not know if the treatment was of significance for the death of the SUD victims in this study, but we know that the medication was given without knowing the vulnerability due to an at that time undiscovered disease.

\section{Autopsy for crime investigation}

Post-mortem DNA analysis of SUD victims opens new diagnostic possibilities but is to our knowledge not yet included in routine investigation in any country. This is probably because these cases often are handled in the juridical/legal system; it is in some countries not legal to do these examinations without permission of the next kin. The main purpose of the forensic autopsy is to investigate possible crimes and only secondary to investigate cause of death due to disease. Furthermore, the analyses have so far been very expensive, especially in the perspective that the investigations are not carried out as part of a medical mission. It is recommended that all SUD victims undergo expert cardiac pathology to rule out a structural heart disease [41]. Collection of blood in an EDTA tube or a blood spot card or a frozen sample of tissue is recommended in cases of SUD and sudden unexpected death in infancy for subsequent DNA analysis/genetic testing [41].

\section{Clinical implication}

If post-mortem genetic testing was to be performed routinely, these autopsy-negative cases could get a diagnosis, firstdegree relatives could have an explanation to the death, and family members could be offered predictive testing. Treatment and surveillance of genotype-positive individuals can reduce morbidity and mortality, and noncarriers would benefit from certain knowledge of not being at risk.

\section{Limits of the study}

Not all genes or exons involved in cardiac ion channel disorder were analyzed. Only about $50 \%$ of the SUD victims were referred for genetic testing. The relatively low participation rate may be due to not considering the molecular diagnosis option immediately at autopsy, but first after all other diagnostic options have failed.

\section{Conclusions}

Our study shows that cardiac-channel focused investigation is of great value in establishing the cause of death in unexplained sudden death, thus supporting the transition of a molecular genetic autopsy into routine clinical practice. In view of what is stated above concerning the primary objective of the investigation of these deaths, it is necessary to establish a partnership between health care and justice; both to implement adequate sampling but also to finance analyses. Furthermore, issues relating to handling of positive test results and privacy problems have to be solved.

Acknowledgments We sincerely thank the family members who participated in this study. We also thank the forensic pathologists for sending samples and tissue. This work was supported by grants from the Northern County Councils Cooperation Committee "Visare Norr", The Heart Foundation of Northern Sweden, and The Medical Faculty at Umeå University, Umeå, Sweden.

Open Access This article is distributed under the terms of the Creative Commons Attribution 4.0 International License (http:// creativecommons.org/licenses/by/4.0/), which permits unrestricted use, distribution, and reproduction in any medium, provided you give appropriate credit to the original author(s) and the source, provide a link to the Creative Commons license, and indicate if changes were made.

\section{References}

1. Wisten A et al (2002) Sudden cardiac death in 15-35-year olds in Sweden during 1992-99. J Intern Med 252(6):529-536

2. Morentin B et al (2001) Incidence and causes of sudden death in persons less than 36 years of age. Med Clin 116(8):281-285

3. Basso C et al (2001) Postmortem diagnosis in sudden cardiac death victims: macroscopic, microscopic and molecular findings. Cardiovasc Res 50(2):290-300

4. Holst AG et al (2010) Incidence and etiology of sports-related sudden cardiac death in Denmark - implications for preparticipation screening. Heart Rhythm 7(10):1365-1371

5. Myerburg R, Castellanos A (1997) Cardiac arrest and sudden cardiac death. In: Braunwald E (ed) Heart Disease: A Textbook of Cardiovascular Medicine. WB Saunders, Philadelphia

6. Tester DJ, Ackerman MJ (2012) The molecular autopsy: should the evaluation continue after the funeral? Pediatr Cardiol 33(3):461470

7. Morentin B, Suarez-Mier MP, Aguilera B (2003) Sudden unexplained death among persons 1-35 years old. Forensic Sci Int 135(3):213-217 
8. Puranik R et al (2005) Sudden death in the young. Heart Rhythm: Off J Heart Rhythm Soc 2(12):1277-1282

9. Neuspiel DR, Kuller LH (1985) Sudden and unexpected natural death in childhood and adolescence. J Am Med Assoc 254(10): 1321-1325

10. Doolan A, Langlois N, Semsarian C (2004) Causes of sudden cardiac death in young Australians. Med J Aust 180(3):110-112

11. Tester DJ, Ackerman MJ (2007) Postmortem long QT syndrome genetic testing for sudden unexplained death in the young. J Am Coll Cardiol 49(2):240-246

12. Ackerman MJ et al (2011) HRS/EHRA expert consensus statement on the state of genetic testing for the channelopathies and cardiomyopathies this document was developed as a partnership between the Heart Rhythm Society (HRS) and the European Heart Rhythm Association (EHRA). Heart Rhythm 8(8):1308-1339

13. Boczek NJ, Tester DJ, Ackerman MJ (2012) The molecular autopsy: an indispensable step following sudden cardiac death in the young? Herzschrittmacherther Elektrophysiol 23(3):167-173

14. Skinner JR et al (2011) Prospective, population-based long QT molecular autopsy study of postmortem negative sudden death in 1 to 40 year olds. Heart Rhythm : Official J Heart Rhythm Soc 8(3): $412-419$

15. Winkel BG et al (2012) The prevalence of mutations in KCNQ1, $\mathrm{KCNH} 2$, and SCN5A in an unselected national cohort of young sudden unexplained death cases. J Cardiovasc Electrophysiol 23(10):1092-1098

16. Wisten A et al (2012) Mutation analysis of cases of sudden unexplained death, 15 years after death: prompt genetic evaluation after resuscitation can save future lives. Resuscitation 83(10):1229-1234

17. Behr ER et al (2008) Sudden arrhythmic death syndrome: familial evaluation identifies inheritable heart disease in the majority of families. Eur Heart J 29(13):1670-1680

18. Tan HL et al (2005) Sudden unexplained death: heritability and diagnostic yield of cardiological and genetic examination in surviving relatives. Circulation 112(2):207-213

19. Allegue $\mathrm{C}$ et al (2011) Prevalence of HCM and long QT syndrome mutations in young sudden cardiac death-related cases. Int J Legal Med 125(4):565-572

20. Napolitano C, Priori SG, Bloise R (1993) Catecholaminergic polymorphic ventricular tachycardia, in GeneReviews, R.A. Pagon, et al., Editors: Seattle (WA)

21. Goldenberg I, Zareba W, Moss AJ (2008) Long QT Syndrome. Curr Probl Cardiol 33(11):629-694

22. Priori SG et al (2002) Clinical and molecular characterization of patients with catecholaminergic polymorphic ventricular tachycardia. Circulation 106(1):69-74

23. Wisten A, Zingmark K (2007) Supportive needs of parents confronted with sudden cardiac death - a qualitative study. Resuscitation 74(1):68-74

24. Campuzano $\mathrm{O}$ et al (2014) The role of clinical, genetic and segregation evaluation in sudden infant death. Forensic Sci Int 242:9-15

25. Semsarian C, Ingles J, Wilde AA (2015) Sudden cardiac death in the young: the molecular autopsy and a practical approach to surviving relatives. Eur Heart $\mathrm{J}$
26. Kumar P, Henikoff S, Ng PC (2009) Predicting the effects of coding non-synonymous variants on protein function using the SIFT algorithm. Nat Protoc 4(7):1073-1081

27. Schwarz JM et al (2010) MutationTaster evaluates disease-causing potential of sequence alterations. Nat Methods 7(8):575-576

28. Tavtigian SV et al (2006) Comprehensive statistical study of 452 BRCA1 missense substitutions with classification of eight recurrent substitutions as neutral. J Med Genet 43(4):295-305

29. Splawski I et al (2000) Spectrum of mutations in long-QT syndrome genes. KVLQT1, HERG, SCN5A, KCNE1, and KCNE2. Circulation 102(10):1178-1185

30. Berge KE et al (2008) Molecular genetic analysis of long QT syndrome in Norway indicating a high prevalence of heterozygous mutation carriers. Scand J Clin Lab Invest 68(5):362-368

31. Kapplinger JD et al (2010) An international compendium of mutations in the SCN5A-encoded cardiac sodium channel in patients referred for Brugada syndrome genetic testing. Heart Rhythm : Off J Heart Rhythm Soc 7(1):33-46

32. Stattin EL et al (2012) Founder mutations characterise the mutation panorama in 200 Swedish index cases referred for Long QT syndrome genetic testing. BMC Cardiovasc Disord 12:95

33. Gladding PA et al (2010) Posthumous diagnosis of long QT syndrome from neonatal screening cards. Heart Rhythm : Official J Heart Rhythm Soc 7(4):481-486

34. Bagnall RD et al. (2014) Exome analysis-based molecular autopsy in cases of sudden unexplained death in the young. Heart Rhythm: Off J Heart Rhythm Soc

35. Wisten A, Messner T (2005) Symptoms preceding sudden cardiac death in the young are common but often misinterpreted. Scand Cardiovasc J 39(3):143-149

36. Tretter JT, Kavey RE (2013) Distinguishing cardiac syncope from vasovagal syncope in a referral population. J Pediatr 163(6):16181623 e1

37. Choi G et al (2004) Spectrum and frequency of cardiac channel defects in swimming-triggered arrhythmia syndromes. Circulation 110(15):2119-2124

38. Tester DJ et al (2005) Pathogenesis of unexplained drowning: new insights from a molecular autopsy. Mayo Clinic proceedings. Mayo Clin 80(5):596-600

39. Tisdale JE et al (2012) Prevalence of QT interval prolongation in patients admitted to cardiac care units and frequency of subsequent administration of QT interval-prolonging drugs: a prospective, observational study in a large urban academic medical center in the US. Drug Saf: Int J Med Toxicol Drug Exp 35(6):459-470

40. Martinez-Raga J et al (2013) Risk of serious cardiovascular problems with medications for attention-deficit hyperactivity disorder. CNS Drugs 27(1):15-30

41. Priori SG et al (2013) HRS/EHRA/APHRS expert consensus statement on the diagnosis and management of patients with inherited primary arrhythmia syndromes: document endorsed by HRS, EHRA, and APHRS in May 2013 and by ACCF, AHA, PACES, and AEPC in June 2013. Heart Rhythm : Off J Heart Rhythm Soc 10(12):1932-1963 